\title{
A MAP FOR THE THICK BEAM-BEAM INTERACTION ${ }^{\dagger}$
}

\section{J. Irwin, T. Chen, Stanford Linear Accelerator Center, Stanford University, Stanford, CA 94309 USA}

We give a closed-form expression for the thick beambeam interaction for a small disruption parameter, as typical in electron-positron storage rings. The dependence on transverse angle and position of the particle trajectory as well as the longitudinal position of collision and the waistmodified shape of the beam distribution are included. Large incident angles, as are present for beam-halo particles or for large crossing-angle geometry, are accurately represented. The closed-form expression is well approximated by polynomials times the complex error function. Comparisons with multi-slice representations show even the first order terms are more accurate than a five slice representation, saving a factor of 5 in computation time.

\section{INTRODUCTION}

S. Krishnagopal and R. Siemann[1] have established that it is essential to retain the finite longitudinal thickness of the beam when simulating the beam-beam interaction. Present beam-beam simulation codes[2] represent the thick interaction as a set of thin beam-beam kicks. Since the thin beam-beam kick for a bi-Gaussian distribution is usually represented by the Erskine-Bassetti[3] formula involving the evaluation of two complex error functions, the calculation is computer intensive and the representation of the thick beam-beam interaction as a set of slices slows down the simulation by a factor roughly equal to the number of slices. To find a thick beam-beam generator, we represent the thin beam-beam kick by an exponential Lie map, and the thick beam-beam interaction by an infinite product of thin beam-beam kicks. We then use the Cambell-Baker-Hausdorff formula to compose this product to a single exponential map.

\section{THE THIN BEAM-BEAM KICK}

S. Kheifets[4] has shown that the potential for the Erskine-Bassetti thin beam-beam kick is given by

$d V\left(x, y, \sigma_{x}^{2}, \sigma_{y}^{2}\right)=d N \frac{2 r_{e}}{\gamma} I\left(x, y, \sigma_{x}^{2}, \sigma_{y}^{2}\right)$

where $\mathrm{dN}$ is the number of electrons in the thin sheet, $\mathrm{r}_{\mathrm{e}}$ is the classical electron radius, $\gamma$ is the Lorentz factor of the particle, and $\mathrm{I}\left(\mathrm{x}, \mathrm{y}, \sigma_{\mathrm{x}}^{2}, \sigma_{\mathrm{y}}^{2}\right)$ is the (dimensionless) integral $I\left(x, y, \sigma_{x}^{2}, \sigma_{y}^{2}\right)=\int_{0}^{\infty} d \lambda \cdot g\left(x, \sigma_{x}^{2}, \lambda\right) \cdot g\left(y, \sigma_{y}^{2}, \lambda\right)$

The function $g\left(x, \sigma_{x}^{2}, \lambda\right)=\frac{1}{\sqrt{\pi}} \cdot \frac{e^{-\frac{x^{2}}{\left(2 \sigma_{x}^{2}+\lambda\right)}}}{\sqrt{2 \sigma_{x}^{2}+\lambda}}$

is a normalized Gaussian distribution of rms width $\left(\sigma_{\mathrm{X}}^{2}+\lambda / 2\right)^{1 / 2}$ and a solution of the diffusion equation,

$\frac{1}{2} \frac{\partial^{2}}{\partial x^{2}} g\left(x, \sigma_{x}^{2}, \lambda\right)=\frac{\partial}{\partial \sigma_{x}^{2}} g\left(x, \sigma_{x}^{2}, \lambda\right) \cdot$

The vertical and horizontal kicks from the potential $\mathrm{dV}$ are given by the Erskine-Basseti formula[3] as

$\Delta y^{\prime}+i \Delta x^{\prime}=d N \frac{4 r_{e}}{\gamma} \frac{\sqrt{\pi}}{\sigma}\left[w\left(z_{1}\right)-e^{-\left(\frac{x^{2}}{2 \sigma_{x}^{2}}+\frac{y^{2}}{2 \sigma_{y}^{2}}\right)} w\left(z_{2}\right)\right]$

where

$\sigma=\sqrt{2\left(\sigma_{x}^{2}-\sigma_{y}^{2}\right)}, \quad z_{1}=\frac{1}{\sigma}(x+i y), \quad z_{2}=\frac{1}{\sigma}\left(\frac{\sigma_{y}}{\sigma_{x}} x+i \frac{\sigma_{x}}{\sigma_{y}} y\right)$

and $w(z)=e^{-z^{2}}\left(1+\frac{2 i}{\pi} \int_{0}^{z} d u \cdot e^{u^{2}}\right)$

is the complex error function. The map for this kick may be represented as an exponential Lie map by[5] $M=e^{-: d V:}$.

\section{THE THICK BEAM-BEAM MAP}

We assume the longitudinal particle density is also Gaussian and given by

$\rho\left(s-s_{0}, \sigma_{s}\right)=\frac{1}{\sqrt{2 \pi \sigma_{s}^{2}}} e^{-\frac{\left(s-s_{0}\right)^{2}}{2 \sigma_{s}^{2}}}$.

The potential for a small slice of thickness ds at position $\mathrm{s}$ will be given by

$d V=\rho\left(s-s_{o}, \frac{\sigma_{s}}{2}\right) d s \frac{2 N r_{e}}{\gamma} I\left(x(s), y(s), \sigma_{x}^{2}(s), \sigma_{y}^{2}(s)\right)$

since the beam distribution as seen by the counter-moving particle is fore-shortened by a factor of 2 . The coordinate $s_{0}=c \tau / 2$ is the location of the center of the bunch as experienced by a counter-moving particle whose time of arrival at the interaction point (IP) is delayed by $\tau$. The map through a set of $\mathrm{n}$ slices is given by

$M=\prod_{j=1}^{n} e^{-\left[: \rho\left(s_{j}-s_{o}, \frac{\sigma_{s}}{2}\right) d s_{j} \frac{2 N r_{e}}{\gamma} I\left(x\left(s_{j}\right), y\left(s_{j}\right), \sigma_{x}^{2}\left(s_{j}\right), \sigma_{y}^{2}\left(s_{j}\right)\right):\right]}$

where $s_{j}$ is the longitudinal position of the center of the slice of width $\mathrm{ds}_{\mathrm{j}}$.

The first-order Cambell-Baker-Hausdorff (CBH) formula can be invoked to obtain, in the limit of very small slices,

$M \approx e^{-: G_{1}:}=\prod_{j=1}^{n} e^{-\left[\frac{2 N r_{e}}{\gamma} \cdot \int d s \cdot \rho\left(s-s_{o}, \frac{\sigma_{s}}{2}\right) \cdot d \cdot I \cdot I\left(x(s), y(s), \sigma_{x}^{2}(s), \sigma_{y}^{2}(s)\right):\right]}$

$\dagger$ Work supported by Department of Energy contracts DE-AC03-76SF00515 and DE-AC05-84ER40150 
The transverse position of the particle may be written $\mathrm{x}(\mathrm{s})$ $=\mathrm{x}_{0}+\left(\mathrm{s}^{\left.-\mathrm{s}_{0}\right)} \mathrm{x}^{\prime} 0\right.$ and $\mathrm{y}(\mathrm{s})=\mathrm{y}_{0}+\left(\mathrm{s}^{\left.-\mathrm{s}_{0}\right)} \mathrm{y}^{\prime} 0\right.$, where $\mathrm{x}_{0}$, $\mathrm{x}^{\prime} 0, \mathrm{y}_{0}$ and $\mathrm{y}^{\prime} 0$ are the particle's position and slope at $\mathrm{s}=\mathrm{s}_{0}$. Also $\sigma_{\mathrm{x}}(\mathrm{s})^{2}=\sigma_{\mathrm{x}}{ }^{2}+\mathrm{s}^{2} \sigma_{\mathrm{x}}, 2$ and $\sigma_{\mathrm{y}}(\mathrm{s})^{2}=\sigma_{\mathrm{y}}{ }^{2}+$ $\mathrm{s}^{2} \sigma_{\mathrm{y}}, 2$ describe the longitudinal shape of the waist with $\sigma_{\mathrm{X}}, \sigma_{\mathrm{X}}, \sigma_{\mathrm{y}}$, and $\sigma_{\mathrm{y}}$, being the rms values of the position and slope of the beam distribution at the interaction point (IP).

The first-order $\mathrm{CBH}$ formula implicitly assumes that the trajectory of the particle is not changed by the interaction, and hence it represents an integrated impulse approximation. The accuracy of this impulse approximation may be estimated by noting that for electron storage rings we have

$$
\frac{\Delta y_{\max }^{\prime}}{\sigma_{y^{\prime}}}=\Delta \theta_{y, \max }=4 \pi \xi_{y} \leq 0.4
$$

where we have taken the beam-beam tune-shifts $\xi_{y}=0.03$. The second-order correction can be estimated through a two-slice approximation as:

$\Delta y_{2}^{\prime}=\Delta y \cdot \frac{\partial}{\partial y}\left[\frac{1}{2} \Delta y_{1}^{\prime}\right] \cong \sigma_{s}\left[\frac{1}{2} \Delta y_{1}^{\prime}\right] \cdot \frac{\partial}{\partial y}\left[\frac{1}{2} \Delta y_{1}^{\prime}\right] \leq \frac{1}{4} \sigma_{s} \Delta y_{1}^{\prime} \cdot \frac{\Delta y_{\max }^{\prime}}{\sigma_{y}}$ from which we deduce that

$$
\frac{\Delta y_{2}^{\prime}}{\Delta y_{1}^{\prime}} \leq \frac{1}{4} \frac{\Delta y_{\max }^{\prime}}{\sigma_{y^{\prime}}} \leq 0.1 \quad \text { implying } \quad \frac{\Delta y_{2}^{\prime}}{\sigma_{y^{\prime}}} \leq 0.04
$$

To achieve better than $4 \%$ accuracy it will be necessary to include second-order effects.

\section{The first-order CBH integral}

To find the generator for the thick beam-beam interaction we must perform the integral in equation (11). If we begin by assuming $x^{\prime} 0=\sigma_{x^{\prime}}=\sigma_{y^{\prime}}=0$ the integral to be performed, after changing the order of the $s$ and $\lambda$ integration, is

$Q_{1}=\int d s \cdot \rho\left(s-s_{0}, \frac{\sigma_{s}}{2}\right) \cdot g\left(y_{0}+\left(s-s_{0}\right) y_{0}^{\prime}, \sigma_{y}^{2}, \lambda\right)$

For simplicity we introduce the variables

$$
\hat{s}=\frac{\left(s-s_{0}\right)}{\left(\frac{\sigma_{s}}{2}\right)} \text { and } \hat{y}_{0}^{\prime}=y_{0}^{\prime} \cdot\left(\frac{\sigma_{s}}{2}\right) \text {. }
$$

We evaluate this integral, in a way that will later allow us to manage the full s dependence, by introducing a translation operator to represent

$g\left(y_{0}+\hat{s} \cdot \hat{y}^{\prime}, \sigma_{y}^{2}, \lambda\right)=e^{\hat{s} \cdot \hat{y}^{\prime} \frac{\partial}{\partial y_{0}}} g\left(y_{0}, \sigma_{y}^{2}, \lambda\right)$.

We combine the $\hat{s}$ dependence of the translation operator $e^{\hat{s} \cdot \hat{y}^{\prime} \frac{\partial}{\partial y_{0}}}$ with the $\hat{s}$ dependence of $\rho(\hat{s}, 1)$ and then complete the square, to obtain $\rho(\hat{s}, 1) \cdot e^{\hat{s} \hat{y}^{\prime} \frac{\partial}{\partial y_{0}}}=\rho\left(\hat{s}-\hat{y}^{\prime} \frac{\partial}{\partial y_{0}}, 1\right) \cdot e^{\frac{1}{2} \hat{y}^{\prime 2} \frac{\partial^{2}}{\partial y_{0}^{2}}}$

The integral over $d \hat{s}$ can now be performed by translating $\rho$ : $\int d \hat{s} \cdot \rho\left(\hat{s}-\hat{y}^{\prime} \frac{\partial}{\partial y_{0}}, 1\right)=\int d \hat{s} \cdot e^{-\hat{y}^{\prime} \frac{\partial}{\partial y_{0}} \frac{\partial}{\partial s}} \rho(\hat{s}, 1)$

$=\int d \hat{s} \cdot \sum \frac{1}{n !}\left(-\hat{y}^{\prime} \frac{\partial}{\partial y_{0}}\right)^{n} \frac{\partial^{n}}{\partial \hat{s}^{n}} \rho(\hat{s}, 1)=1$

Hence we have

$$
Q_{1}=e^{\frac{1}{2} \hat{y}^{\prime 2} \frac{\partial^{2}}{\partial y_{0}^{2}}} \cdot g\left(y_{0}, \sigma_{y}^{2}, \lambda\right)
$$

Since $\mathrm{g}$ is a solution of the diffusion equation

$Q_{1}=e^{\hat{y}^{\prime 2} \frac{\partial^{2}}{\partial \sigma_{y}^{2}}} \cdot g\left(y_{0}, \sigma_{y}^{2}, \lambda\right)=g\left(y_{0} \cdot \sigma_{y}^{2}+\hat{y}^{\prime 2}, \lambda\right)$

This result for $\mathrm{Q}_{1}$ implies that the thick beam-beam potential for the case $\mathrm{x}^{\prime} 0=\sigma_{\mathrm{X}}{ }^{\prime}=\sigma_{\mathrm{y}}{ }^{\prime}=0$ is just the original potential with $\sigma_{y}^{2}$. replaced by $\sigma_{y}^{2}+\hat{y}^{\prime 2}$.

Next we perform the integral with only $\sigma_{\mathrm{X}}{ }^{\prime}=\sigma_{\mathrm{y}}{ }^{\prime}=0$. $Q_{2}=\int d \hat{s} \cdot \rho(\hat{s}, 1) \cdot g\left(x_{0}+\hat{s} \hat{x}^{\prime}, \sigma_{x}^{2}, \lambda\right) \cdot g\left(y_{0}+\hat{s} \hat{y}^{\prime}, \sigma_{y}^{2}, \lambda\right)$

$$
=e^{\hat{x}^{\prime} \hat{y}^{\prime}} \frac{\partial}{\partial x_{0}} \frac{\partial}{\partial y_{0}} g\left(x_{0}, \sigma_{x}^{2}+\hat{x}^{\prime 2}, \lambda\right) \cdot g\left(y_{0}, \sigma_{y}^{2}+\hat{y}^{\prime 2}, \lambda\right)
$$

The integral with only $\sigma_{\mathrm{X}}$, $=0$ is:

$Q_{3}=\int d s \cdot \rho\left(s-s_{0}, \frac{\sigma_{s}}{2}\right) \cdot g\left(x_{0}+\left(s-s_{0}\right) x_{0}^{\prime}, \sigma_{x}^{2}, \lambda\right)$.

$$
\cdot g\left(y_{0}+\left(s-s_{0}\right) y_{0}^{\prime}, \sigma_{y}^{2}+s^{2} \sigma_{y^{\prime}}^{2}, \lambda\right)
$$

In changing $\mathrm{s}$ to $\hat{S}$ it is convenient to introduce $\hat{s}_{0}={\frac{s_{0}}{\left(\frac{\sigma_{s}}{2}\right)}}^{\prime} \sigma_{\hat{y}^{\prime}}=\frac{\sigma_{s}}{2} \sigma_{y^{\prime}}$ and $\sigma_{y_{0}}^{2}=\sigma_{y}^{2}+s_{0}^{2} \sigma_{y^{\prime}}^{2}$.

Using these definitions

$g\left(y_{0}+\hat{s} \hat{y}^{\prime}, \sigma_{y_{0}}^{2}+\sigma_{\hat{y}^{\prime}}^{2}\left(2 \hat{s}_{0} \hat{s}+\hat{s}^{2}\right), \lambda\right)$

$\left.=e^{\frac{\sigma_{\hat{y}^{\prime}}^{2}}{2}\left(2 \hat{s}_{0}\right.} \frac{\partial}{\partial \hat{y}^{\prime}} \frac{\partial}{\partial y_{0}}+\frac{\partial^{2}}{\partial \hat{y}^{\prime 2}}\right) e^{\hat{s} \hat{y}^{\prime} \frac{\partial}{\partial y_{0}}} g\left(y_{0}, \sigma_{y_{0}}^{2}, \lambda\right)$

The first factor has no $\hat{s}$ dependence and can be taken out of the integral. The remaining integral, after translating $\mathrm{x}_{0}$, is just $\mathrm{Q}_{2}$. We can now write down an expression for the double integral in the generator of eq. 11 with no assumptions on x'0, y'0, $\sigma_{\mathrm{X}}$ ', and $\sigma_{\mathrm{y}}$ ':

$$
\begin{aligned}
& G_{1}= \frac{2 N r_{e}}{\gamma} e^{\frac{\sigma_{\hat{x}^{\prime}}^{2}}{2}\left(2 \hat{s}_{0} \frac{\partial}{\partial \hat{x}^{\prime}} \frac{\partial}{\partial x_{0}}+\frac{\partial^{2}}{\left.\partial \hat{x}^{\prime 2}\right)}\right.} e^{\frac{\sigma_{\hat{y}^{\prime}}^{2}}{2}\left(2 \hat{s}_{0}\right.} \frac{\partial}{\partial \hat{y}^{\prime}} \frac{\partial}{\partial y_{0}}+\frac{\partial^{2}}{\left.\partial \hat{y}^{\prime 2}\right)} e^{\hat{x}^{\prime} \hat{y}^{\prime}} \frac{\partial}{\partial x_{0}} \frac{\partial}{\partial y_{0}} \\
& I\left(x_{0}, y_{0}, \sigma_{x_{0}}^{2}+\hat{x}^{\prime 2}, \sigma_{y_{0}}^{2}+\hat{y}^{\prime 2}\right)
\end{aligned}
$$

\section{First-order symplectification}

To obtain a symplectic evaluation of $\mathrm{G}_{1}$ one can introduce a mixed-variable generator

$F_{1}\left(x, y, X^{\prime}, Y^{\prime}\right)=x X^{\prime}+y Y^{\prime}+G_{1}\left(x, y, X^{\prime}, Y^{\prime}\right)$.

The implicit equations can be solved approximately by expanding $\mathrm{F}_{1}$ in a Taylor series about the point $\left(\mathrm{x}, \mathrm{x}^{\prime}, \mathrm{y}, \mathrm{y}^{\prime}\right)$.

\section{Evaluation of the derivatives of the generator}

The calculation can be arranged so that the derivatives of $\mathrm{G}_{1}$ required to compute coordinate changes can be found from derivatives of the complex error function. Derivatives of $I\left(x_{0}, y_{0}, \sigma_{x_{0}}^{2}+\hat{x}^{\prime 2}, \sigma_{y_{0}}^{2}+\hat{y}^{\prime 2}\right)$, with 
respect to $\hat{x}^{\prime}$ or $\hat{y}^{\prime}$ may be replaced by derivatives with respect to $\sigma_{\mathrm{x} 0} 0^{2}$ and $\sigma_{\mathrm{y} 0} 0^{2}$ which may in turn be replaced by derivatives with respect to $\mathrm{x}_{0}$ and $\mathrm{y}_{0}$. Thus the computation reduces to derivatives of the Erskine-Bassetti formula with respect to $\mathrm{x}_{0}$ and $\mathrm{y}_{0}$. These derivatives become polynomials times the complex error function by using the relationship

$\frac{\partial w(z)}{\partial z}=-2 z w(z)+\frac{2 i}{\sqrt{\pi}}$

The order of this polynomial depends on the accuracy with which the waist effects are represented. The largest corrections to the thin beam-beam kick, arising from the slope y', are accounted for by replacing $\sigma_{\mathrm{y}}^{2}$ by $\sigma_{y}^{2}+\hat{y}^{\prime 2}$.

\section{The second-order CBH integral}

The second order CBH may also be computed. The result is

$G_{2}=\frac{1}{2}\left(\frac{2 N r_{e}}{\gamma}\right)^{2} \frac{1}{\sqrt{\pi}}\left[\left(\frac{\partial I}{\partial y}\right)^{2}+\hat{y}^{\prime 2}\left(\frac{\partial^{3} I}{\partial y^{3}} \frac{\partial I}{\partial y}-\left(\frac{\partial^{2} I}{\partial y^{2}}\right)^{2}\right)+\ldots\right]$

where $I=I\left(x, y, \sigma_{x}^{2}, \sigma_{y}^{2}+\frac{\hat{y}^{\prime 2}}{2}\right)$.

$\mathrm{G}_{2}$ can be inserted into equation

$$
\begin{gathered}
F_{12}\left(x, y, X^{\prime}, Y^{\prime}\right)=x X^{\prime}+y Y^{\prime}+G_{1}\left(x, y, X^{\prime}, Y^{\prime}\right) \\
+\frac{1}{2} \frac{\partial G_{1}\left(x, y, X^{\prime}, Y^{\prime}\right)}{\partial x} \frac{\partial G_{1}\left(x, y, X^{\prime}, Y^{\prime}\right)}{\partial X^{\prime}} \\
+\frac{1}{2} \frac{\partial G_{1}\left(x, y, X^{\prime}, Y^{\prime}\right)}{\partial y} \frac{\partial G_{1}\left(x, y, X^{\prime}, Y^{\prime}\right)}{\partial Y^{\prime}}
\end{gathered}
$$

to obtain a second order implicit generator.

\section{NUMERICAL RESULTS}

We compare the results calculated by the thick beambeam interaction with the multiple slice approach for the vertical plane, since the horizontal plane has little thick beam-beam effect. We scanned the range of $y=0$ to $10 \sigma$, $y^{\prime}=-10$ to $10 \sigma$, in $1 / 4 \sigma$ steps. In Table 1 , the maximum and r.m.s. differences from a 21 -slice results are listed.

The thick beam-beam integral may be calculated for various orders. The 1 st order includes the lowest order of correction of $\mathrm{G}_{1}$ - up to the 1st order derivatives of single beam-beam kick, and the 5 th order includes up to 5 th order derivatives. Then, 2 nd order $\mathrm{CBH}$ correction, $\mathrm{G}_{2}$, is included respectively. Finally, both 2nd order Poisson Bracket of $\mathrm{G}_{1}$ and $\mathrm{CBH}$ corrections are included.

One can see that even the 1st order thick beam-beam calculation gives better result than 5 slices. Notice that in this approximation, the $y^{\prime}$ kick requires essentially the same computation time as a single slice, implying a factor of 5 can be saved in beam-beam simulation. In addition, the maximum errors occur at about $1 \sigma$ in thick beam-beam, unlike the multi-slices methods which have larger error at

\begin{tabular}{|c|c|c|c|c|c|}
\hline & & \multicolumn{2}{|c|}{$\begin{array}{c}\text { location of } \\
\text { maximum } \\
\text { error }(\sigma)\end{array}$} & \multicolumn{2}{|c|}{$\begin{array}{c}\text { Errors: }{ }^{*} \\
\left(\Delta \mathrm{z}-\Delta \mathrm{z}_{21}\right) / \max \left(\Delta \mathrm{z}_{21}\right) \\
\mathrm{z}=\mathrm{y} \text { or } \mathrm{y}^{\prime}\end{array}$} \\
\hline & & $\mathrm{y}$ & $\mathrm{y}^{\prime}$ & $\max (\%)$ & r.m.s. $(\%)$ \\
\hline \multirow[t]{2}{*}{1 slice } & $\mathrm{y}$ & 0 & 9.25 & 100 & .032 \\
\hline & $\mathrm{y}^{\prime}$ & 1.75 & -10 & 60 & .233 \\
\hline \multirow[t]{2}{*}{3 slices } & $\mathrm{y}$ & 4.5 & -10 & 34 & .009 \\
\hline & $\mathrm{y}^{\prime}$ & 4.75 & -10 & 14 & .05 \\
\hline \multirow[t]{2}{*}{5 slices } & $\mathrm{y}$ & 5.75 & -10 & 20 & .005 \\
\hline & $\mathrm{y}^{\prime}$ & 5.75 & -10 & 7.4 & .024 \\
\hline \multirow{2}{*}{$\begin{array}{l}\text { thick BB } \\
1 \text { st order }\end{array}$} & $\mathrm{y}$ & 1.5 & -1.5 & 4.9 & .001 \\
\hline & $\mathrm{y}^{\prime}$ & 1 & -1 & 5.1 & .017 \\
\hline \multirow{2}{*}{$\begin{array}{l}\text { thick BB } \\
5 \text { th order }\end{array}$} & $\mathrm{y}$ & .75 & 5.5 & 5.5 & .002 \\
\hline & $\mathrm{y}^{\prime}$ & 1 & 1.25 & 3.5 & .015 \\
\hline \multirow{2}{*}{$\begin{array}{c}\text { thick BB } \\
\text { w/ 2nd } \mathrm{CBH}^{\dagger}\end{array}$} & $\mathrm{y}$ & 2 & 4.75 & 4.9 & .002 \\
\hline & $\mathrm{y}^{\prime}$ & 1.25 & 1.75 & 3.0 & .015 \\
\hline \multirow{2}{*}{$\begin{array}{c}\text { thick BB } \\
\text { w/2ndPB\& } \mathrm{CBH}^{\dagger}\end{array}$} & $\mathrm{y}$ & 7.75 & 10 & 4.8 & .001 \\
\hline & $\mathrm{y}^{\prime}$ & 1.25 & 1.75 & 3.0 & .014 \\
\hline
\end{tabular}
large $y^{\prime}$.

Table 1. Comparison of beam-beam kick with $s_{0}=0$

$* \mathrm{z}_{21}$ indicates the $\Delta \mathrm{z}$ calculated with a 21-slice method.

$\dagger$ These results should be considered as preliminary.

\section{CONCLUSION}

We represent the thick beam-beam interaction as the infinite product of thin kicks. When the disruption parameter is small it is valid to compose this product to a single map defined by a generator given by the $\mathrm{CBH}$ formula. The largest effect by far in typical beam-beam situations is the dependence on the vertical slope. The first order computation for this case results in the replacement of $\sigma_{\mathrm{y}}^{2}$ by $\sigma_{\mathrm{y}}^{2}+\left(\sigma_{\mathrm{s}} / 2 \mathrm{y}^{\prime}\right)^{2}$ in the Erskine-Bassetti formula The numerical comparison shows the thick beam-beam method, especially the 1st order approach, can replace the multi-slice method which has been used in most of beambeam simulations, and save substantial CPU time.

\section{REFERENCES}

[1]. S. Krishnogopal and R. Siemann, Phys. Rev. D 41, 2312 (1990).

[2]. T. Chen, J. Irwin and R. Siemann, Physical Review E, Vol. 49, No. 3, p2323, March 1994; A. Zholents and DN Shatilov, this proceeding; J. L. Tennyson, ABC29, (1991).

[3]. Erskine-Bassetti, CERN-ISR-TH/80-06 (1980).

[4]. S. Kheifets, PETRA NOTE 119,DESY(Jan., 1976)

[5]. J. Irwin, SLAC-PUB-6713, submitted to Nuclear Instruments and Methods (1995). 